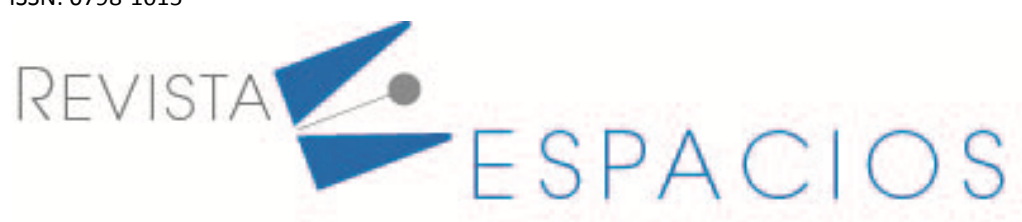

\title{
Modelos pedagógicos aplicados en educación inicial
}

\section{Pedagogical models applied in early education}

\author{
ROMAN, Jimy V. ${ }^{1}$ \\ PEÑAFIEL RODRÍGUEZ, Miriam P. ${ }^{2}$ \\ ALVEAR, Luis F. ${ }^{3}$ \\ CHAVEZ, Roberto C. ${ }^{4}$ \\ VINUEZA, Mishel E. ${ }^{5}$
}

\begin{abstract}
Resumen
La presente investigación analizó la aplicación de metodologías y modelos pedagógicos en el nivel inicial en las instituciones educativas de la ciudad de Riobamba, Ecuador, en el periodo: enero de 2019septiembre de 2020. A través de un enfoque mixto, la investigación aplicada, exploratoria, descriptiva, explicativa, no experimental y longitudinal obtuvo como resultados que, en su mayoría, las maestras de Educación Inicial utilizan el método constructivista en función de lograr un aprendizaje significativo y un desarrollo integral.

Palabras clave: metodología, modelos pedagógicos, inicial
\end{abstract}

\begin{abstract}
In this research, the methodologies applied at the initial level in educational institutions of the city of Riobamba in the period 2019-2020 have been analyzed. Using a mixed methodology, that is, qualitative and quantitative. With a type of applied, exploratory, descriptive, explanatory, non-experimental and longitudinal research. Obtaining as results that most of the initial education teachers use the constructivist method, thus achieving significant learning and integral development.

key words: Methodology, Pedagogical models, Initial
\end{abstract}

\section{Introducción}

El Modelo Pedagógico en la Educación Inicial usualmente se asocia con el diseño y ejecución de estrategias, metodologías y herramientas que hagan posible y visible la acción de maestros y estudiantes en el proceso de formación. Estos van desde el nivel inicial hasta la escuela, con el propósito de llevar a cabo una metodología que facilite este proceso, con actividades que lo trasladen fuera del ámbito académico hacia la comunidad. (Gutiérrez, 2013)

\footnotetext{
${ }^{1}$ Docente. Carrera de Educación Inicial. Universidad Nacional de Chimborazo. jroman@unach.edu.ec 2 Docente. Carrera de Educación Inicial. Universidad Nacional de Chimborazo.mpenafiel@unach.edu.ec 3 Docente. Carrera de Educación Inicial. Universidad Nacional de Chimborazo. luis.alvear@unach.edu.ec 4Docente. Carrera de Educación Inicial. Universidad Nacional de Chimborazo.rchavez@unach.edu.ec 5Estudiante. Carrera de Educación Inicial. Universidad Nacional de Chimborazo. mvinueza@unach.edu.ec
} 
Al constituirse la educación como un fenómeno social, los modelos pedagógicos resultan propios de la reflexión en torno a la educación, reconocida no solo como un saber sino también como un objeto de crítica conceptual y de revisión de los fundamentos sobre los cuales se haya construido. (Rómulo, 1990)

Según De Zubiría (2010), los textos y cuadernos escolares, así como el material didáctico, la forma de disponer el salón o simplemente el mapa o el recurso didáctico utilizado, nos dicen mucho más de los enfoques pedagógicos de lo que aparentemente podría pensarse. Son en realidad la huella inocultable de nuestra concepción pedagógica. Se entiende que en la anterior idea las prácticas cotidianas del aula de clase son las manifestaciones materiales de un modelo pedagógico que de manera implícita se encuentra en las acciones didácticas de los maestros. Es decir, que el docente posee un discurso teórico-implícito que da soporte a sus prácticas de enseñanza.

Existen numerosos modelos pedagógicos que han explicado las diferentes concepciones, metodologías y técnicas a lo largo de la historia. Muchos de ellos aún se practican en nuestro quehacer educativo, de ellos citamos:

Modelo tradicional: tuvo su auge en los siglos XVI y XVII, se caracteriza por estar centrado en la enseñanza y en la consecuente transmisión de información, por lo que el rol protagónico pertenece al educador. La comunicación es unidireccional, excluye a los estudiantes y sus experiencias o saberes previos; a quienes corresponde escuchar y repetir los conceptos ofrecidos por el profesor. Caracterizado, además, como un modelo autoritario, memorístico, acrítico, repetitivo y poco o nada reflexivo, se aplica a la lectura y a la copia. Una clase magistral que permita la transmisión de conocimientos es considerada eficiente.

Modelo Conductista: en Latinoamérica se desarrolló durante los años 60, propone una manera eficiente, estandarizada y rápida de educación a partir del condicionamiento, a través del cual el estudiante adaptaba las conductas e ideas que el planificador había establecido. La metodología en este modelo se basa en la fijación de aprendizajes mediante el refuerzo y la instrucción. El educando es activo en cuanto a la ejecución de acciones programadas, pero pasivo en cuanto a la recepción de contenidos, por lo que el docente ejecuta un plan preestablecido.

Modelo activo: llamado también Escuela Nueva o Pedagogía de la Acción, sus representantes principales son: John Dewey, Ovidio Decroly, María Montessori y Celestin Freinet. La Escuela Nueva como corriente pedagógica reformadora trata de cambiar el rumbo de la educación tradicional y libresca, para darle un sentido activo al proceso educativo, al basarse en los principios de respeto a la individualidad del niño en sus aptitudes y capacidades para que pueda desarrollar lo mejor de sí mismo; de educar al individuo para la convivencia y cooperación social; de globalización de la enseñanza como un todo organizado con un criterio unitario y totalizador; y de autoeducación, considera al estudiante como el centro de toda actividad escolar.

Modelo Constructivista: plantea que el aprendizaje es una construcción personal de quien aprende y la tarea de aprender cobra sentido en la medida que admiten las personas a construirse y apropiarse del mundo.

Los conocimientos no se adquieren, sino se construyen al interior de los sujetos, hecho que permite el desarrollo de las capacidades intelectuales. El constructivismo fue desarrollado a principios del siglo XX producto del aporte de muchos pensadores, y dio lugar a varias corrientes. Pasados los años 80 se desarrolla una fuerte tendencia por integrar estas posturas epistemológicas, incorporando los aspectos más representativos de cada una de ellas; así tenemos la teoría psicogenética de Piaget (Arias-Arroyo, Merino-Zurita y Peralvo-Arequipa, 2017), la psicosocial de Vigotsky (Vielma y Salas, 2000) y el aprendizaje significativo de Ausubel (Viera, 2003), principales representantes del constructivismo.

Modelo Pedagogía Conceptual: se atribuye a los hermanos Zubiría Samper ser los innovadores de este modelo. La Pedagogía Conceptual está orientada al desarrollo de la inteligencia en todas sus manifestaciones, presenta 
como propósito fundamental formar seres humanos amorosos, éticos, talentosos, creadores, competentes expresivamente, es decir, en un solo término: analistas simbólicos. (De Zubiría, 2010)

Modelo didáctico: es una herramienta teórico-práctica con la que se pretende transformar una realidad educativa, orientada hacia los protagonistas del hecho pedagógico como lo son estudiantes y docentes. Para Socarrás, Díaz y Sáez (2012) un modelo didáctico es una construcción teórico formal que centrada en fundamentos científicos e ideológicos, intenta interpretar el proceso de enseñanza-aprendizaje y dirigirlo hacia determinados fines educativos. Todo modelo didáctico se desprende de un modelo pedagógico y orienta las actuaciones concretas del docente en el aula.

La aplicación de instrumentos de recolección de datos (ficha de observación y encuesta) en el nivel inicial de las instituciones objeto de estudio, identificó la aplicación de metodologías pedagógicas provenientes de los enfoques conductista, constructivista y espontáneo.

\section{Metodología}

\subsection{Diseño de la investigación}

El diseño de la investigación es no experimental porque se realizó sin manipular las variables. Se basó fundamentalmente en la observación del fenómeno tal y como se da en el contexto educativo para analizarlo después. Es descriptiva porque permitió determinar las características fundamentales de los involucrados en el proceso. Es documental porque se realizó a través de la consulta de documentos (libros, revistas, periódicos, memorias, anuarios, registros, códices, constituciones, entre otros). Es mixta porque implica combinar los enfoques cualitativos y cuantitativos en un mismo estudio.

\subsection{Método, técnica e instrumento}

Se utilizó el método de la observación directa, así también la técnica de la encuesta y su correspondiente instrumento: el cuestionario, aplicado a los docentes de Educación Inicial de las unidades educativas bajo estudio, situadas en la ciudad de Riobamba, en el periodo enero de 2019-septiembre de 2020.

\subsection{Población y muestra}

\subsubsection{Población}

La población fue no aleatoria, conformada por 12 unidades educativas y 1 municipal, de las cuales 6 son fiscales, 2 son fisco-misionales, 1 municipales y 4 particulares:

Tabla 1

Tipos de Instituciones participantes

en la Investigación

\begin{tabular}{c|c|c} 
Tipo de institución & No & F\% \\
\hline Fiscal & 6 & $59 \%$ \\
\hline Municipales & 1 & $2 \%$ \\
\hline Particulares & 4 & $27 \%$ \\
\hline Fisco-misionales & 2 & $12 \%$ \\
\hline total & 13 & $100 \%$ \\
\hline
\end{tabular}

Fuente: Encuesta

Elaboración: elaboración propia 
Muestra: No se seleccionó una muestra, dado que el estudio es descriptivo, por lo que se trabajó con toda la población.

\subsubsection{Instrumentos}

Ficha de Observación y Cuestionario; este último contó con preguntas claves, precisas y objetivas relacionadas con los objetivos e indicadores de cada variable de estudio.

\section{Resultados:}

Tabla 2

Instituciones participantes en la investigación

Nombre de la institución

Centro de Desarrollo Infantil Municipal $n^{\circ} 4$

Centro de Educación Inicial "Politécnico"

Centro de Educación Inicial "Alfredo Costales Cevallos"

Centro de Educación Inicial Ángel Guardián

Centro Infantil Juan Pablo II

Oxford Gardens School

Unidad Educativa Simón Rodríguez

Unidad Educativa Cristiana Nazareno

Centro de Educación Inicial Safari Kids

Unidad Educativa Liceo Policial de Chimborazo

Unidad Educativa Fiscomisional San Vicente de Paúl

Unidad Educativa "Combatientes de Tapi"

Unidad Educativa "Riobamba"

TOTAL: 13 Instituciones Educativas

Fuente: Información recolectada

Elaboración: propia

\subsection{Análisis e interpretación}

Considerando que dentro de la provincia de Chimborazo esta conformada por ditritos para la presente investigación se tomó en cuenta a 13 instituciones educativas del Distrito Riobamba - Chambo, haciendo énfasis a las instituciones de Educación Inicial, y donde nuestros estudiantes de la carrera de Educación Inicial, realizan sus practicas cuirriculares, ejecución 1 y ejecución 2, siendo estas instituciones de acuerdo al tipo de mantenimiento las siguientes: fiscal, fiscomisional, municipal y particular. 
Tabla 3

Aplicación del modelo pedagógico constructivista.

\begin{tabular}{|c|c|c|c|}
\hline Nombre de la Institución & Si & No & Ocasionalmente \\
\hline Centro de Desarrollo Infantil Municipal $n^{\circ} 4$ & & $\mathrm{X}$ & \\
\hline Centro de Educación Inicial "Politécnico" & & & $X$ \\
\hline Centro de Educación Inicial "Alfredo Costales Cevallos" & & $\mathrm{x}$ & \\
\hline Centro de Educación Inicial Ángel Guardián & $x$ & & \\
\hline Centro Infantil Juan Pablo II & & $\mathrm{x}$ & \\
\hline Oxford Gardens School & $x$ & & \\
\hline Unidad Educativa Simón Rodríguez & & $\mathrm{X}$ & \\
\hline Unidad Educativa Cristiana Nazareno & $x$ & & \\
\hline Centro de Educación Inicial Safari Kids & & $\mathrm{X}$ & \\
\hline Unidad Educativa Liceo Policial de Chimborazo & & & $x$ \\
\hline Unidad Educativa Fiscomisional San Vicente de Paúl & & & $x$ \\
\hline Unidad Educativa "Combatientes de Tapi" & & $\mathrm{X}$ & \\
\hline Unidad Educativa "Riobamba" & & $\mathrm{x}$ & \\
\hline TOTAL & 3 & 7 & 3 \\
\hline
\end{tabular}

Fuente: encuesta

Elaboración: propia

Gráfico 1

Aplicación del modelo Pedagógico Constructivista

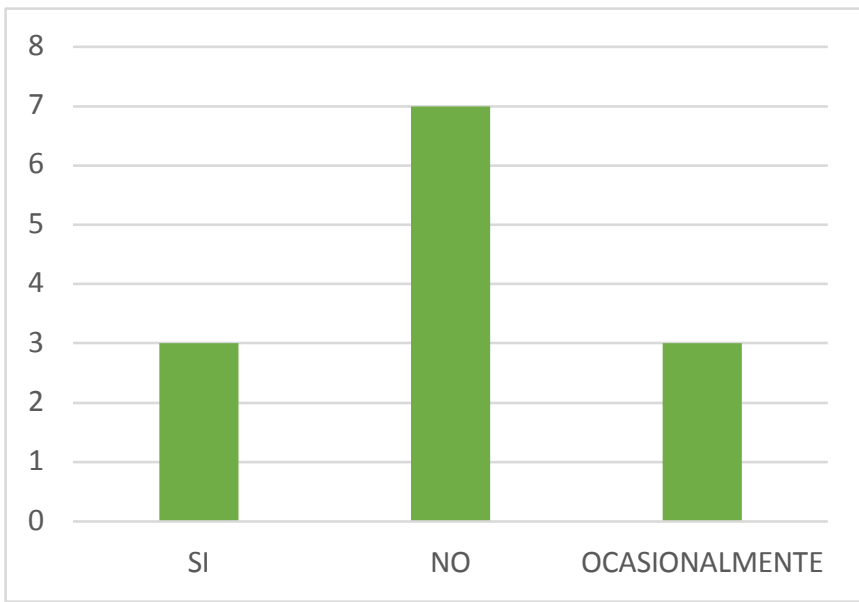

Fuente: encuesta

Elaboración: propia

De acuerdo a la información podemos darnos cuenta que 7 Instituciones fiscales son aquellas que no aplican el modelo Pedagógico Constructivista en el Proceso de Ensenanza aprendizaje con los niños y niñas de educación inicial en cambio existe una similitud entre las instituciones particulares y fiscomisionales al aplicar el modelo pedagógico constructivista. 
Tabla 4

Capacitación a los Docentes por Institución sobre los modelos Pedagógicos.

\begin{tabular}{|c|c|c|c|}
\hline Nombre de la institución & SI & NO & A VECES \\
\hline Centro de Desarrollo Infantil Municipal $n^{\circ} 4$ & & $X$ & \\
\hline Centro de Educación Inicial "Politécnico" & & $X$ & \\
\hline Centro de Educación Inicial "Alfredo Costales Cevallos" & & & $\mathrm{X}$ \\
\hline Centro de Educación Inicial Ángel Guardián & & $\mathrm{X}$ & \\
\hline Centro Infantil Juan Pablo II & & $X$ & \\
\hline Oxford Gardens School & $X$ & & \\
\hline Unidad Educativa Simón Rodríguez & & $\mathrm{X}$ & \\
\hline Unidad Educativa Cristiana Nazareno & & & $\mathrm{X}$ \\
\hline Centro de Educación Inicial Safari Kids & $\mathrm{X}$ & & \\
\hline Unidad Educativa Liceo Policial de Chimborazo & & & $\mathrm{X}$ \\
\hline Unidad Educativa Fiscomisional San Vicente de Paúl & & & $\mathrm{X}$ \\
\hline Unidad Educativa "Combatientes de Tapi" & & $\mathrm{X}$ & \\
\hline Unidad Educativa "Riobamba" & & $\mathrm{X}$ & \\
\hline TOTAL & 2 & 7 & 4 \\
\hline
\end{tabular}

Gráfico 2

Capacitación a los docentes por Institución sobre los modelos pedagógicos

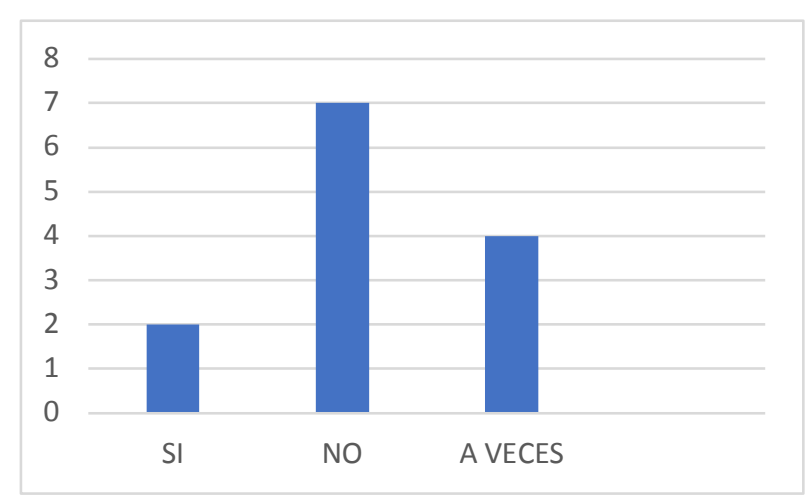

Fuente: encuesta

Elaboración: propia

De las 13 instituciones encuestadas, 2 reciben capacitación de Modelos Pedagógicos, en cambio 7 no reciben capacitación alguna de los modelos los mismos que son indispensables dentro del curriculo, y 4 instituciones a veces reciben capacitación y es unicamente al inicio del año.

El ministerio de Educación a través de sus planes y programas y acorde al curriculum establecido sugiere que todo docente debe recibir la capacitación necesaria para el perfeccionamiento docente y este debe ser mucho más si se trabaja en el nivel inicial, puesto que responde a los resultados de aprendizaje, sin embargo se puede observar que existe falencias en el desarrollo evolutivo de los infantes, produciendo así este desface en su proceso de formación y la poca aplicación de teorias y modelos. 
Deduciendo que estas instituciones por contar con los recursos económicos son más accesibles a las capacitaciones a sus docentes y proveer el material didáctico.

Tabla 5

Relación con los modelos y el nivel educativo

\begin{tabular}{c|c|c} 
Nivel educativo & Modelo aplicado & Frecuencia \\
\hline INICIAL 1 & Conductista & 2 \\
\hline & Constructivista & 1 \\
\hline INICIAL 2 & Espontaneo & 1 \\
\hline & Conductista & 6 \\
\hline & Constructivista & 1 \\
\hline Total, general & Espontaneo & 2 \\
\hline
\end{tabular}

Fuente: encuesta

Elaboración: propia

Gráfico 3

Métodos que se ocupan en cada nivel educativo.

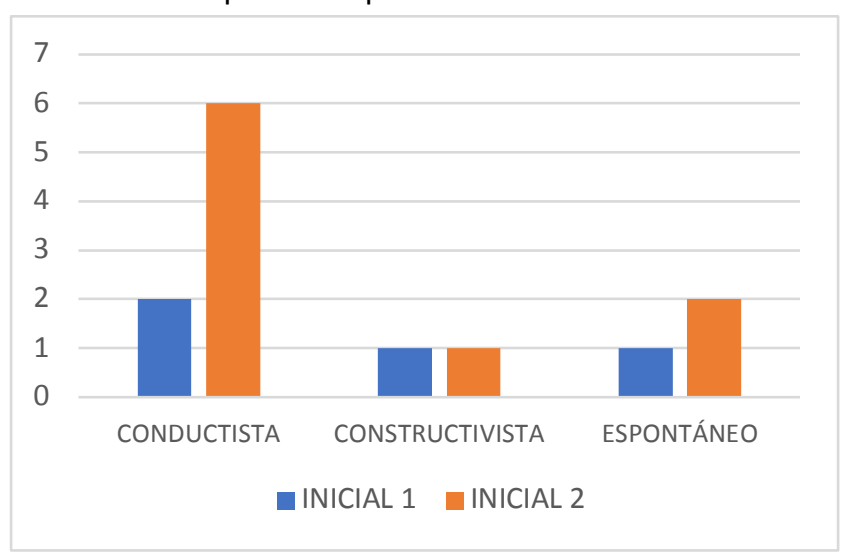

Fuente: encuesta

Elaboración: propia

En la gráfica 3, respondieron a los métodos que se ocupan cada nivel, en el inicial 1, el método conductista, el método constructivista y el método espontáneo han ocupado el $17 \%$ y en el inicial 2 , el método conductista, el método constructivista y el método espontáneo han ocupado el $16 \%$. 
Tabla 6

Relación por institución según ficha de observación (aplicación de los Modelos Pedagógicos)

Etiquetas de fila

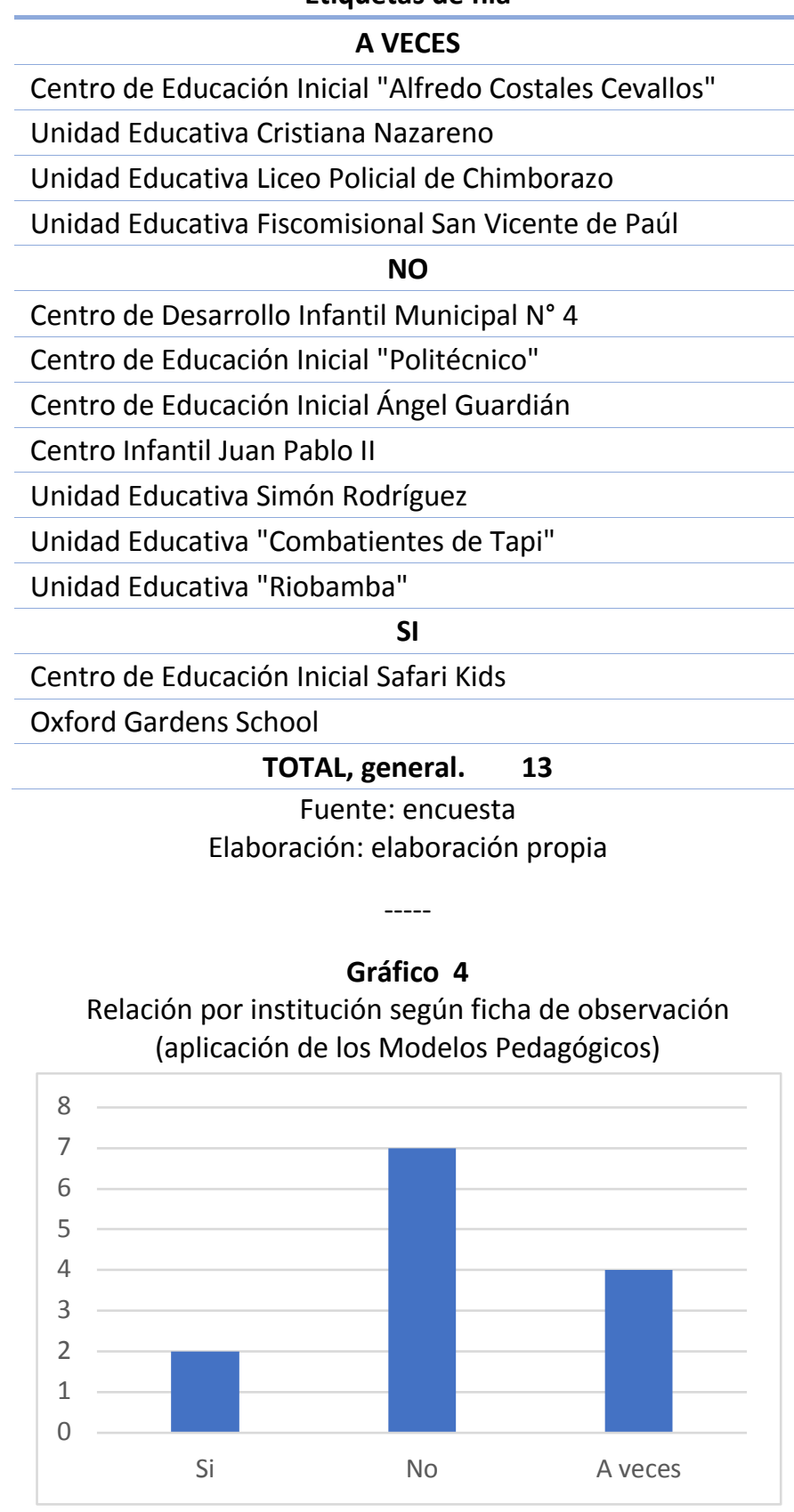

Fuente: encuesta

Elaboración: elaboración propia

En el gráfico 4, de los 13 centros de Desarrollo Infantil donde se aplico las Fichas de Observación 2 instituciones SI aplican los Modelos Pedagógicos, 7 instituciones NO aplican los Modelos Pedagógicos y 4 instituciones aplican A VECES los Modelos Pedagógicos que son necesarios para un desarrollo adecuado en el proceso de aprendizaje de los estudiantes. 
Tabla 7

Relación por institución según ficha de observación

Fase No. 4 (evaluación y aplicación)

Etiquetas de fila

\begin{tabular}{l}
\hline APLICA \\
\hline Unidad Educativa Liceo Policial de Chimborazo \\
\hline Unidad Educativa Fiscomisional San Vicente de Paúl \\
\hline Centro de Educación Inicial Safari Kids \\
\hline Oxford Gardens School \\
\hline Centro de Educación Inicial "Alfredo Costales Cevallos" \\
\hline Unidad Educativa Cristiana Nazareno \\
\hline Centro de Desarrollo Infantil Municipal N 4 \\
\hline Centro de Educación Inicial "Politécnico" \\
\hline Centro de Educación Inicial Ángel Guardián \\
\hline Centro Infantil Juan Pablo II \\
\hline Unidad Educativa Simón Rodríguez \\
\hline $\begin{array}{r}\text { Unidad Educativa "Combatientes de Tapi" } \\
6,8\end{array}$ \\
\hline Unidad Educativa "Riobamba" \\
\hline Fuente: ficha de observación \\
Elaboración: propia
\end{tabular}

En el gráfico 5, se puedo detectar de acuerdo a las fichas de observación aplicadas en las 13 instituciones educativas, que 6 instituciones realizan lo que es la evaluación y la aplicación de todo el proceso que conlleva una planificación de clase adecuada para que se la pueda ejecutar de una mejor manera, y 7 instituciones no la aplican.

\section{Conclusiones}

La aplicación de los modelos pedagógicos tradicional, conductista, activo o constructivista en las instituciones bajo estudio resulta aleatoria, por lo que no existe preferencia hacia un modelo en específico. Dentro de los 
contextos educativos actuales, marcados por la implementación de reformas a gran escala, la oferta de programas de desarrollo profesional de alta calidad propone cambios en los modelos pedagógicos que solidifiquen e incorporen principios que ayuden a los estudiantes.

Los modelos pedagógicos son métodos de aprendizaje orientados a una visión educativa en los estudiantes, ya que se hacen responsables de su propio aprendizaje y logran aplicar sus habilidades y conocimientos adquiridos en el salón de clases, basándose en sus actitudes, experiencias y conocimientos preexistentes, al relacionarse con el modelo pedagógico de la planificación, ya que le dan sostén a la comprensión y sentido de un nuevo aprendizaje.

La relación de los modelos pedagógicos dentro de la planificación curricular tiene la necesidad de transformar las necesidades de las instituciones y mejorar, ya que la planificación permite unir una determinada teoría pedagógica con la práctica docente, con el fin de mejorar la calidad de los modelos pedagógicos con actividades desarrolladas en las aulas, talleres y laboratorios de las instituciones, ya que la educación que reciben los niños es fundamental para su desarrollo cognitivo, emocional, social y motriz, y tiene un impacto que trasciende su infancia y favorece, a lo largo de su vida, a sus familias y a la sociedad.

\section{Referencias bibliográficas}

Arias-Arroyo, P. A., Merino-Zurita, M. M. y Peralvo-Arequipa, C. R. (2017). Análisis de la Teoría de Psicogenética de Jean Piaget: Un aporte a la discusión. Dom. Cien., 3(3), 833-845.

De Zubiría, J. (2010). A refundar la escuela. México: Asociación de Educadores de Latinoamérica y El Caribe.

Gutiérrez. (2013). Modelos Pedagógicos y Estrategias Didácticas. Colombia: Basedoc.

Rómulo, G. B. (1990). Saber Pedagogía. Santa Fe de Bogotá: Mesa Redonda Magisterio.

Socarrás, S., Díaz, M. y Sáez, A. (2012). El Profesor Guía: máximo orientador del trabajo educativo en la Educación Médica Superior Cubana. Humanidades Médicas, 12(3), 427- 446.

Vielma, E. y Salas, M. L. (2000). Aportes de las teorías de Vygotsky, Piaget, Bandura y Bruner. Paralelismo en sus posiciones en relación con el desarrollo. Educere, 3(9), 30-37.

Viera, T. (2003). El aprendizaje verbal significativo de Ausubel. Algunas consideraciones desde el enfoque histórico cultural. Universidades, 26, 37-43.

Esta obra está bajo una Licencia Creative Commons Attribución-NoCommercial 4.0 International

(cc) EY-NC 